\title{
C.O.S - 3DRA - Software construtor de objetos tridimensinais: \\ Uma proposta de Construção de Sólidos Geométricos Através da Realidade Aumentada
}

\section{COS - 3DRA - Software constructor of 3d objects: \\ A Proposal of Construction of Solid Geometry through an Augmented Reality}

\author{
Rodolfo Gregório de Moraes ${ }^{1}$ \\ Carlos Vitor de Alencar Carvalho ${ }^{2}$ \\ Ana Maria Severiano de Paiva ${ }^{3}$
}

\section{Palavras-chave:}

Novas tecnologias aplicadas ao ensino

Realidade aumentada

Construções geométricas espaciais.

\section{Resumo}

Numa era em que as tecnologias invadem a vida dos cidadãos, em especial nas áreas urbanas, como podemos aumentar a capacidade de percepção espacial e mesmo investigar as transformações geométricas, se apropriando de recursos tecnológicos? Neste artigo objetivamos responder este questionamento com a exploração de construções de sólidos geométricos, através da combinação de sólidos previamente determinados, a saber, o cubo, o cone, a esfera e o cilindro, através da mediação da Realidade Aumentada (RA). Essa manipulação dos sólidos favorece a evolução gradual do conhecimento geométrico do aluno/usuário que, devido à forma lúdica apresentada, baseada em modelos pré-organizados que o desafia, faz com que este reflita sobre suas ações de forma crítica, percebendo, através de erros na construção, a necessidade de implementação de determinada ação corretiva para obter o êxito na atividade proposta.

\begin{abstract}
In an era that technologies invade the lives of citizens, especially in urban areas, how can we increase the capacity of spatial perception and even investigate the geometric transformations, appropriating technology resources? This article aims to answer this question by the exploration of geometric solids constructions, through a combination of solids defined as box, cone, sphere and cylinder through the mediation of the Augmented Reality. This manipulation of solids favors the gradual evolution of geometrical knowledge of the student / user, that due to the playfully way presented based on pre-arranged models that challenge, make him/ her reflect upon his/her actions in a critical way, noting through errors in the construction the necessity to implement specific corrective action to achieve success in the proposed activity.
\end{abstract}

Key Words:

New technologies applied to education,

Augmented reality

Space geometric constructions

\section{Artigo \\ Original \\ Original \\ Paper}

Recebido em 03/2011

Aprovado em $04 / 2011$

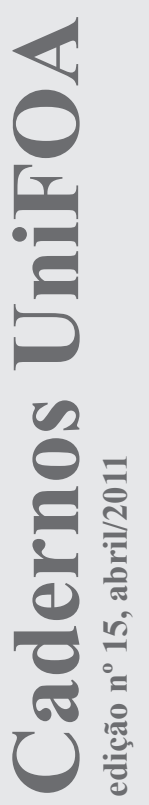

\footnotetext{
${ }^{1}$ Docente da Fundação Educacional de Duque de Caxias - FEUDUC. Discente do Programa de Mestrado Profissional em Educação Matemática USS (Vassouras - Rio de Janeiro - Brasil).

${ }^{2}$ Docente do Programa de Pós-Graduação - Mestrado Profissional em Educação Matemática da Universidade Severino Sombra - USS (Vassouras - Rio de Janeiro - Brasil). Docente do UniFOA (Volta Redonda - Rio de Janeiro - Brasil). Docente do Instituto Superior de Tecnologia - FAETEC (Paracambi - Rio de Janeiro - Brasil)

${ }^{3}$ Docente do Programa de Pós-Graduação - Mestrado Profissional em Educação Matemática da Universidade Severino Sombra (Vassouras - Rio de Janeiro - Brasil).
} 


\section{Introdução}

O processo de transformação- ocorrido na sociedade devido ao desenvolvimento e implementação de ferramentas tecnológicas, em especial, os computadores e ferramentas atreladas à utilização deste, como a Internetdesafia concepções e práticas do profissional de educação. Percebemos que iniciativas de inserção das tecnologias no ensino vêm ocorrendo no Brasil desde a década de 70, do século $\mathrm{XX}$, e deram origens a projetos como o EDUCOM, PRONINFE e PROINFO, coordenados pelo Ministério da Educação MEC. Não podemos imaginar que somente a existência de uma política pública voltada à inserção da tecnologia ao ensino é suficiente para mudar as práticas docentes. Contudo, não podemos negar que, em um mundo cada vez mais tecnológico, os processos de ensino-aprendizagem não se modifiquem. Essa relação dialética entre a sociedade e a escola é bem observada por Chervel (1990) que cita que o processo de criação de uma disciplina se transforma em uma via de mão dupla, visto que a escola sofre influências da sociedade bem como, tem o poder de gerar transformações na sociedade em que está inserida.

Segundo Valente (1999, p. 39), a escola é um espaço de trabalho complexo, que envolve inúmeros outros fatores, além do professor e dos alunos. "[...] Portanto, a mudança na escola deve envolver todos os participantes do processo educativo - alunos, professores, diretores, especialistas, comunidade de pais".

Cabe ressaltar o papel do professor nesse novo cenário, pois aquele deixará de ser o profissional responsável pela informação de conteúdos de determinada área de conhecimento, mas acompanhará o desenvolvimento do aluno em seu processo de solução de problemas e geração de conhecimento. Para a intervenção efetiva, não existe uma receita, e o que é ser efetivo é polêmico, pois depende de um contexto teórico, das concepções do professor e das limitações culturais e sociais que se apresentam em uma determinada situação. Esses fatores nunca são exatamente os mesmos, variando de um ambiente para o outro e para cada aluno no mesmo ambiente (VALENTE, 1999). Ou seja, conforme citado por Chervel (1990) o único limite verdadeiro com o qual se depara a liberdade pedagógica do mestre é o grupo de alunos que ele encontra diante de si.

Nesse contexto, este trabalho apresenta o desenvolvimento de um software com tecnologia de Realidade Aumentada, chamado COS 3DRA (Constructor Objects Software 3D RA). O software foi desenvolvido em linguagem $\mathrm{C}$ e, para a construção dos sólidos básicos, utilizou o sistema gráfico OpenGL (WOO, 1999) que através da intermediação da RA permita ao usuário tentar configurar um sólido semelhante ao desafio proposto através da combinação de 4 sólidos básicos disponíveis para seleção, a saber: A Cubo, O Cone, O Cilindro e A Esfera, sendo possível ao aluno/ usuário, também, alterar as dimensões destes sólidos básicos, rotacioná-los e transladá-los

\section{O Ensino da Geometria}

A geometria está presente em todos os momentos de nossa vida, claro que as atitudes que fazemos diariamente, até certo ponto, podem ser consideradas intuitivas, ou seja, aplicamos conhecimentos matemáticos sem a preocupação de estarmos "calculando" algo. Para ilustrar melhor, imaginemos uma pessoa que precisa posicionar uma pequena mesa retangular numa área livre de uma sala. Essa pessoa não fará medições formais para calcular a área livre e as dimensões da mesa para verificar se é possível posicionar a mesa neste local, ela efetuará uma comparação e, se considerar possível tentará posicionar a mesa na área livre. Esse exemplo simples nos faz pensar: "então, por qual razão devemos estudar Geometria?"

Conforme citado no PCN para o Ensino Fundamental (1998), os conceitos geométricos constituem parte importante do currículo de Matemática "[...] por meio deles, o aluno desenvolve um tipo especial de pensamento que lhe permite compreender, descrever e representar, de forma organizada, o mundo em que vive".

Segundo Van Hiele (PONTE e SERRAZINA, apud MORELATTI, 2006, p. 267), o pensamento geométrico evolui articulando a intuição e a dedução de forma progressiva em uma sequência de cinco níveis de compreensão de conceitos, sendo que a passagem de um nível para o seguinte ocorre através de vivência 
de atividades adequadas e ordenadas. Essa passagem entre os níveis depende mais da aprendizagem do que idade e maturação do aluno.

Cada nível é caracterizado por uma linguagem própria e uma relação entre os objetos de estudo. No primeiro nível, as formas são reconhecidas por suas aparências, ainda sem preocupação com propriedades, nível da visualização. No segundo nível, as propriedades de uma figura são reconhecidas e usadas na resolução de problemas, nível da análise. No terceiro nível, é possível estabelecer inter-relações entre figuras, ordenando logicamente as propriedades de determinada figura, nível da ordenação. No quarto nível, temos o domínio do processo dedutivo de demonstrações geométricas, nível da dedução. E, no quinto nível, temos a capacidade de compreender demonstrações formais, nível do rigor.

Ainda em relação ao ensino de geometria o estudo dos registros de representações semiótica de Raymond Duval (DUVAL, apud ALMOULOUD, et al, 2004, p. 99), no que se refere as dificuldades de compreensão dos conceitos, cita que existem os seguinte tipos de apreensão durante os processos de ensino e de aprendizagem:

a) sequencial: é solicitada nas tarefas de construção ou nas de descrição com objetivo de reproduzir uma figura;

b) perceptiva: é a interpretação das formas da figura em uma situação geométrica;

c) discursiva: é a interpretação dos elementos da figura geométrica, privilegiando a articulação dos enunciados através da imersão dos mesmos numa rede semântica de propriedades do objeto;

d) operatória: é uma apreensão centrada nas modificações possíveis de uma figura e na reorganização perceptiva que essas modificações sugerem.

Assim, na resolução de problemas de geometria podemos classificar os tipos de modificações possíveis de uma figura (ibid):

a) modificação "mereológica": a figura pode decompor-se em subfiguras, fracionando-se e reagrupando-se con- venientemente segundo uma relação parte-todo;

b) modificação ótica: é a transformação de uma figura em outra, que será denominada "imagem";

c) modificação posicional: é o deslocamento da figura em relação a um referencial.

O nosso interesse no presente trabalho consiste neste último processo de modificação (modificação posicional), que pode ser realizado graficamente ou manualmente, em nosso caso, através do software C.O.S. - 3D R.A. baseado em conceitos de R.A, realizado coerentemente de acordo com o nível de percepção geométrica do aluno, segundo a teoria de Van Hiele, pois a combinação destes níveis de modificações permite o fracionamento de uma figura, a combinação de uma figura com outras figuras, gerando formas mais complexas ampliando o seu domínio dos conceitos geométricos e percepção espacial

\section{Realidade Virtual e Aumentada}

A Realidade Virtual (RV) possui diversas possibilidades de aplicação, nas mais variadas áreas, dentre as quais podemos destacar Medicina, Entretenimento, Arquitetura, Engenharia e Educação. Na Medicina, pode-se imaginar a utilização de luvas interativas para realização de cirurgias a distância; no entretenimento, já convivemos com os filmes 3-D e diversos jogos eletrônicos que possibilitam a imersão em mundos virtuais; na arquitetura, temos a possibilidade de realizar um passeio virtual por um projeto de construção; na engenharia, criação de protótipos de peças, equipamentos e testes em simulações de situações reais; e em educação, a criação de micromundos, para realização de experiências físicas, é um pequeno exemplo do potencial de utilização da RV.

O que vamos enfatizar dentre as mais variadas formas de aplicação da RV é o seu potencial pedagógico para a educação matemática. Uma das áreas da RV, que podemos utilizar com apoio ao ensino de matemática, é a Realidade Aumentada (RA). Esse termo é uma subclassificação dentro de um concei- 
to de RV- chamado Realidade Misturada, na qual predomina a fusão do ambiente real com o ambiente virtual, gerado por computador (ZORZAL et al, 2006). Assim, de acordo com a predominância do tipo de ambiente, podemos ter uma Virtualidade Aumentada, em que predominam os elementos virtuais e uma Realidade Aumentada, caso haja a predominância de elementos reais.

A possibilidade de manipulação de objetos virtuais num ambiente real é um dos principais diferenciais da RA, pois, estimula o usuário a usar a tecnologia devido a interatividade flexibilizada. Assim, o usuário amplia seu poder de visualização num ambiente real de objetos gerados num mundo virtual, contudo, faz-se necessário o uso de uma biblioteca que seja capaz de fazer essa mediação entre o ambiente real e os objetos virtuais, posicionado corretamente a partir de instruções feitas pelo usuário via teclado.

Dentre algumas bibliotecas disponíveis, destaca-se o Artoolkit (ARTOOLKIT, 2010), que é um conjunto de bibliotecas desenvolvidas na linguagem $\mathrm{C}$ pelo laboratório de Interface Humana da Universidade de Washington, que tem como objetivo auxiliar o programador na construção rápida de aplicações na área de RA. Disponível gratuitamente para fins não comerciais, o pacote disponibilizado contém bibliotecas para rastreamento e os códigos fonte dessas bibliotecas para permitir que programadores adaptem para as suas necessidades.

Um dos principais problemas encontrados na implementação de aplicativos em RA é o cálculo preciso do ponto de vista do usuário em tempo real, de modo que as imagens virtuais estejam alinhadas com os objetos reais. A biblioteca ARToolKit permite que imagens virtuais sejam sobrepostas ao vivo no mundo real. Ao optarmos neste artigo pela utilização da RA através do ARToolkit, estamos cientes tanto do potencial dessa técnica, quanto das limitações inerente ao software, como por exemplo, a necessidade de uma proximidade do marcador em relação ao computador.

\section{A proposta do software}

Este artigo traz a proposta de criação de um aplicativo, desenvolvido em linguagem C e, para a construção dos sólidos básicos, utilizou-se o sistema gráfico OPENGL (WOO, 1999) que através da intermediação da RA permita ao usuário tentar configurar um sólido semelhante ao desafio proposto através da combinação de 4 sólidos básicos disponíveis para seleção, a saber: A Caixa, O Cone, O Cilindro e A Esfera, sendo possível ao aluno/ usuário, também, alterar as dimensões deste sólidos básicos, rotacioná-los e transladá-los.

A construção desse sólido, o qual foi gerado por essa combinação, é uma tarefa que requer do aluno/usuário, além de uma percepção espacial, uma reflexão sobre como utilizar os comandos do aplicativo. A atividade a definição de uma sequência de transformações tridimensionais para que o sólido gerado seja o esperado e, conforme citado por Valente (1999), esse processo desencadeia o ciclo Descrição-Execução-Depuração, que é de grande relevância para o conhecimento, pois permite que o aluno/usuário se torne crítico em relação aos comandos selecionados. É comum em uma atividade desse tipo que erros ocorram, que não devem ser encarados como punitivos, mas uma possibilidade de verificação das falhas e correções dos comandos. Para exemplificar a proposta, foi construído um exemplo conforme a Figura 1. Nela é indicado o desafio proposto. O aluno/usuário deve selecionar as formas básicas necessárias e as transformações geométricas para chegar próximo do desafio. Isso deve ser feito através de comandos do para cada tipo de transformação: Escala, Rotação no eixo X, Y e Z, translação no eixo $\mathrm{X}, \mathrm{Y}$ e Z).

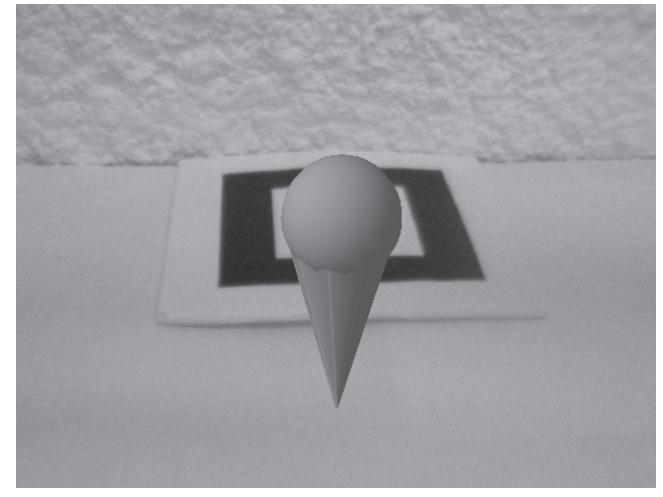

Figura 1 - Desafio a ser construído. 
Na versão inicial deste protótipo, os comandos executados pelo aluno/usuário devem ser anotados detalhadamente para posterior verificação pelo docente do procedimento adotado para alcançar o objetivo, permitindo assim uma avaliação do tipo de percepção geométrica do aluno e possíveis falhas no processo de aprendizagem, bem como a reflexão desse docente em relação a sua ação pedagógica. Como demonstração do potencial desse aplicativo, efetuamos uma sequência de comandos conforme telas a seguir.

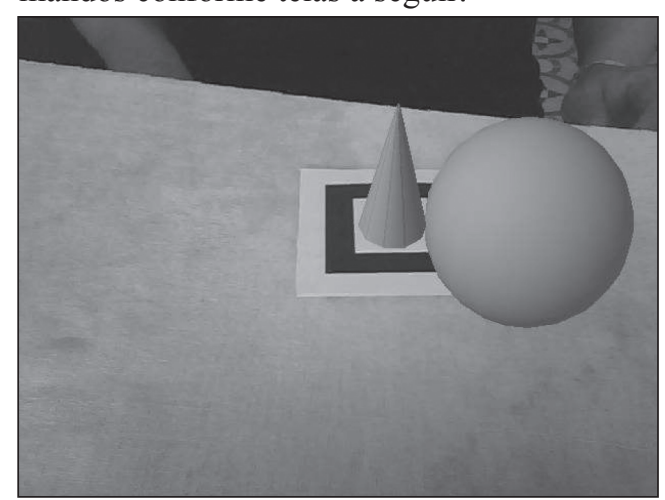

Figura 2 - Seleção das formas básicas iniciais no software.

A figura 2 ilustra a seleção das formas básicas iniciais necessárias para resolver o desafio proposto na figura 1 , em sua configuração original, de acordo com o padrão préestabelecido no aplicativo C.O.S - 3.D. R.A. A partir desse ponto, o aluno/usuário inicia $\mathrm{o}$ processo de comandos geradores das transformações geométricas. A figura 3 apresenta a redução do raio da esfera.

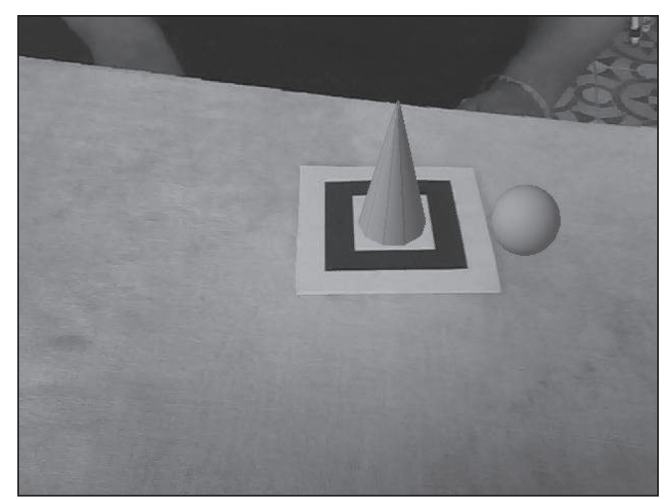

Figura 3 - Transformação da esfera Redução do Raio através da escala.

A seguir as figuras 4 e 5 demonstram a transformação de rotação efetuada no cone, as quais nomearemos de posição 1 , e 2 .

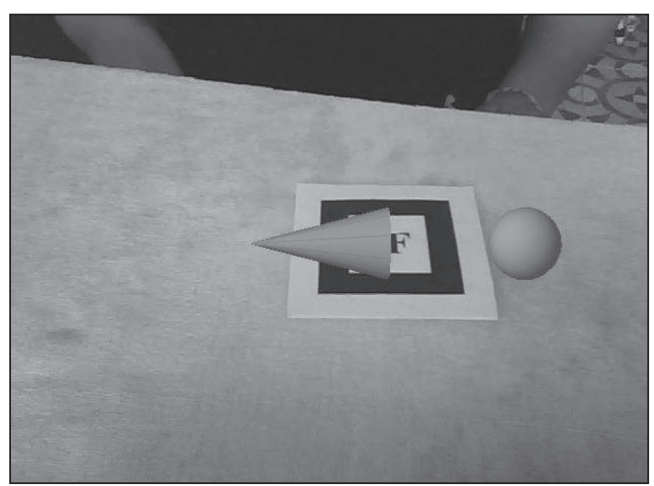

Figura 4 - Transformação do cone - Rotação axial posição 1

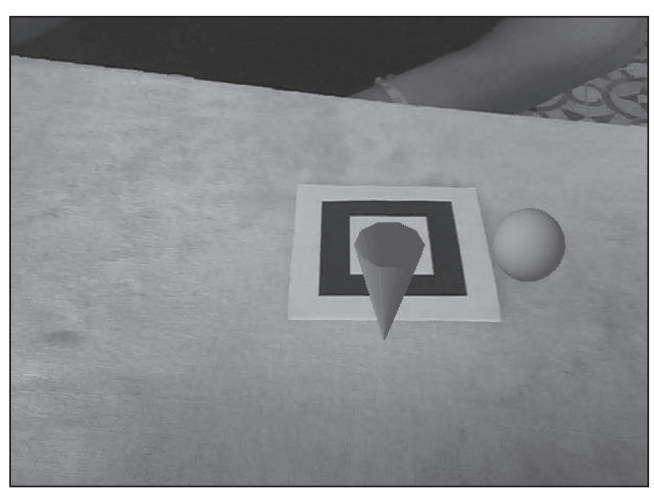

Figura 5 - Transformação do cone - Rotação axial posição 2

Agora, com o cone devidamente posicionado, vamos posicionar a esfera, alinhando verticalmente com o cone, conforme as figuras 6 e 7 .

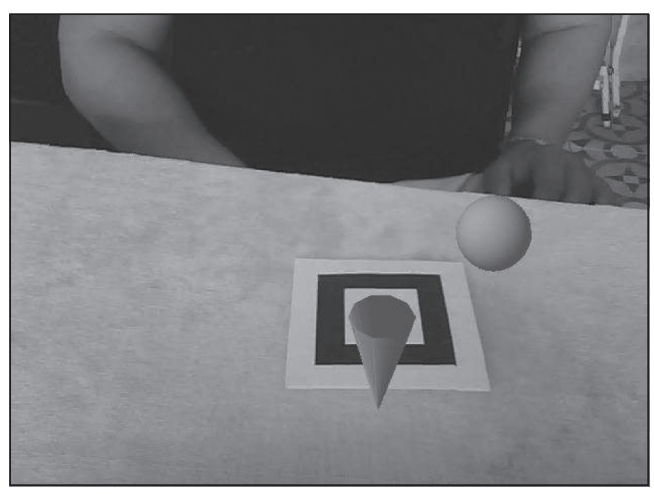

Figura 6 - Transformação do cone Translação para alinhamento vertical

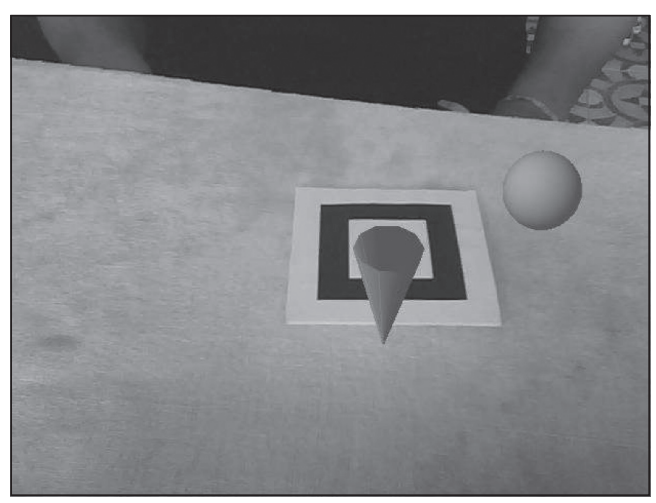

Figura 7-Transformação do cone-Translação para alinhamento vertical. 
Depois do alinhamento vertical basta deslocar de encontro ao cone, conforme as figuras 8 e 9 .

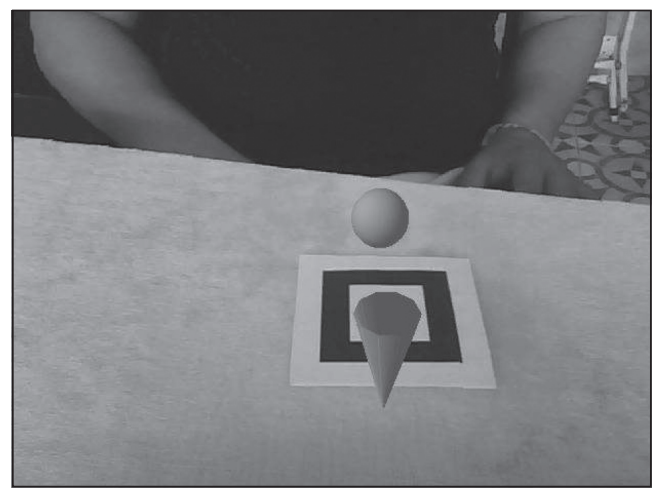

Figura 8 - Transformação da esfera - Translação para alinhamento final.

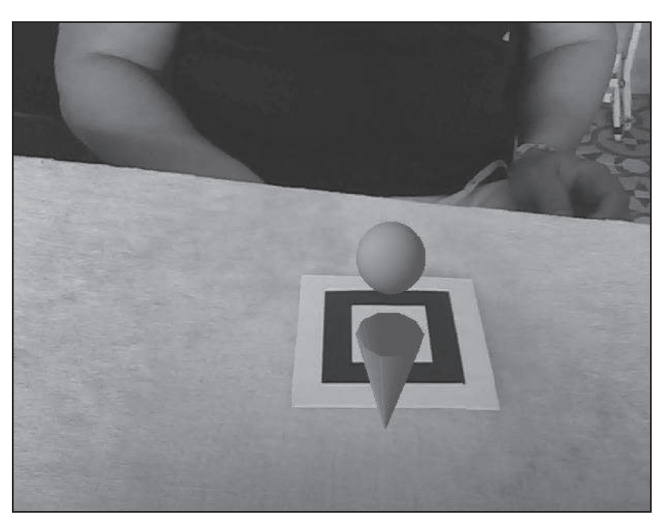

Figura 9 - Transformação da esfera - Translação para alinhamento final

Por fim obtemos o resultado esperado, ou seja, uma figura similar àquela proposta no desafio inicial (figura 10).

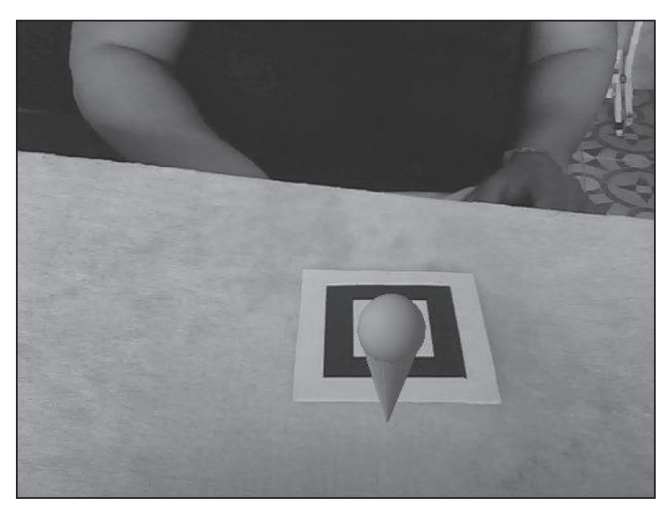

Figura 10 - Sólido gerado - Resultado final

\section{Considerações finais}

O objetivo que pretendemos alcançar com o desenvolvimento do Software Construtor de Objetos 3.D - C.O.S - 3.D. R.A. é disponibilizar uma ferramenta que pode ser aplicada ao ensino de matemática como forma de flexibilizar a percepção de caracterís- ticas individuais de sólidos elementares, bem como o efeito de comandos de transformações geométricas como homotetias e, translações e rotações em torno de eixos cartesianos para a construção de sólidos gerados pela combinação dos sólidos elementares.

O potencial de utilização dessa ferramenta para o profissional de educação deve estar alinhado com o planejamento do trabalho educativo, permitindo a exploração gradual dos desafios, de acordo com o perfil dos alunos envolvidos nas atividades. Como todo processo de pesquisa experimental, as críticas construtivas- baseadas em relatos de experiência- são de grande valia para a evolução do trabalho e aprimoramento da ferramenta, logo, os autores se mostram, desde já, disponíveis para debates que levem a melhoria da qualidade do ensino de matemática. Novos desafios estão sendo propostos no software para posterior utilização/validação do mesmo em sala de aula.

\section{Agradecimentos}

A terceira autora agradece à FAPERJ pelo apoio financeiro através do programa "apoio à melhoria do ensino em escolas sediadas no estado do Rio de Janeiro - 2009". O segundo autor agradece ao CNPq pelo apoio financeiro através da Bolsa de Produtividade em Desenvolvimento Tecnológico e Extensão Inovadora - DT.

\section{Referências bibliográficas:}

ALMOULOUD, Saddo Ag et al. A geometria no ensino fundamental: reflexões sobre uma experiência de formação envolvendo professores e alunos. Rev. Bras. Educ., Dez 2004, no.27, p.94-108. ISSN 1413-2478

ARTOOLKIT ARTOOLKIT versão 2.72. Disponível em: http://www.hitl.washington. edu/artoolkit/documentation/index.html. Acesso em: 01/12/2010

BRASIL, Ministério da Educação. Parâmetros Curriculares Nacionais: Ensino Fundamental - Matemática. Brasília: Secretaria de Ensino Fundamental, 1998. 
CAMELO, Marcelo A. Ambiente em Realidade Virtual para usuários de Educação a Distância : Estudo da Viabilidade Técnica. Florianópolis, 2001. Dissertação de Mestrado (Mestrado em Engenharia da Produção) Universidade Federal de Santa Catarina;

CANTARELLI, Elisa Maria Pivetta Software Educacional II- Pós-Graduação Especialização em Informática na Educação - Universidade Regional Integrada do Alto Uruguai e das Missões - 2004

CARRARD, Marcos. Tutorial VRML. Ijuí, 2001. Universidade Regional do Noroeste do Estado do Rio Grande do Sul- UNIJUÍ

CHERVEL, André. História das disciplinas escolares: reflexões sobre um campo de pesquisa. Teoria e Educação, Porto Alegre, $\mathrm{n}^{\circ}$ 2 177-229, 1990.

FOSSE, Juliana Moulin. Representação Cartográfica Interativa Tridimensional: Estudo da variável cor em ambiente VRML. Curitiba, 2004. Dissertação de Mestrado
(Mestrado em Ciências Geodésicas) Universidade Federal do Paraná.

MANSSOUR, Isabel Harb . Introdução á VRML 2.0. Porto Alegre, RS PUC/ RS Disponível em http://www.inf.pucrs. br/ manssour/VRML/index.html - Acessado em 30 de Outubro de 2010.

MORELATTI, Maria Raquel Miotto; SOUZA, Luís Henrique Gazeta de. Aprendizagem de conceitos geométricos pelo futuro professor das séries iniciais do Ensino Fundamental e as novas tecnologias. Curitiba, Educar, n.28, p. 263-275, 2006. Editora UFPR

VALENTE, José Armando. O Computador na sociedade do conhecimento. Campinas, SP UNICAMP/NIED, 1999.

ZORZAL, E. R.; BUCCIOLI, Arthur Augusto Bastos; KIRNER, Cláudio. Usando Realidade Aumentada no Desenvolvimento de Quebracabeças Educacionais. In: SVR2006 - VIII Symposium on Virtual Reality, 2006, BelémPA. Proceedings of VIII Symposium on Virtual Reality. Belém, PA : Editora CESUPA, 2006. p. 221-232.

\section{Endereço para Correspondência:}

Carlos Vitor de Alencar Carvalho

cvitorc@gmail.com

Centro Universitário de Volta Redonda

Campus Três Poços

Av. Paulo Erlei Alves Abrantes, $n^{\circ}$ 1325,

Três Poços - Volta Redonda / RJ

CEP: $27240-560$

Informações bibliográficas:

Conforme a NBR 6023:2002 da Associação Brasileira de Normas Técnicas (ABNT), este texto científico publicado em periódico eletrônico deve ser citado da seguinte forma: MORAES, Rodolfo Gregório de; CARVALHO, Carlos Vitor de Alencar; PAIVA, Ana Maria Severiano de C.O.S - 3DRA - Software construtor de objetos tridimensinais: Uma proposta de Construção de Sólidos Geométricos através da Realidade Aumentada Cadernos UniFOA. Volta Redonda, Ano VI, n. 15, abril 2011. Disponível em: <http://www. unifoa.edu.br/cadernos/edicao/15/29.pdf> 